\title{
The harmonic bandwidth of phase-reversal discrimination
}

\author{
PATRICK J. BENNETT \\ University of Toronto, Toronto, Ontario, Canada
}

\begin{abstract}
Previous studies have shown that $180^{\circ}$ relative phase shifts in $f+2 f$ gratings are discriminated when the cosine or the sine component of the shift exceeds some criterion (Bennett \& Banks, 1987; Field \& Nachmias, 1984). The current experiments demonstrate that this result holds for other two-component gratings, provided that the components are within two to three octaves of each other. For frequency differences greater than two to three octaves, phase-reversal discrimination is impossible. A simple model that discriminates phase shifts on the basis of changes in the responses of even- and odd-symmetric spatial filters can account for the results.
\end{abstract}

Spatial phase is an important source of information for pattern recognition and identification (Oppenheim \& Lim, 1981; Piotrowski \& Campbell, 1982). Recent studies have shown that human observers are remarkably sensitive to spatial phase, at least in foveal vision (Badcock, 1984a, 1984b; Burr, Morrone, \& Spinelli, 1989), but the mechanisms used to discriminate spatial phase shifts are poorly understood. One reason for our lack of understanding is that phase shifts are associated with multiple changes in the spatial arrangement of features in an image. Even in relatively simple patterns, such as two-component sinewave gratings, phase shifts can alter overall (peak-totrough) and local contrast, luminance maxima or minima, the magnitude of luminance gradients, and the width and skewness of individual bars. This multiplicity of cues raises the problem of establishing which cue, or which combination of cues, is used to discriminate phase shifts. Addressing this problem is made more difficult by the fact that not all cues are always available, and there is as yet no consensus on the relative utility of these cues across discrimination tasks. One potential solution to this problem is to adopt a more abstract representation of spatial phase that is less dependent on the particular spatial characteristics of a pattern. The current paper examines the utility of such an approach that was first described by Field and Nachmias (1984).

Field and Nachmias (1984) measured sensitivity to phase shifts in $f+2 f$ compound gratings by fixing the contrast of the fundamental at some suprathreshold value and varying the contrast of the second harmonic until a

Portions of this work were presented at the 1990 meeting of the Association for Research in Vision and Ophthalmology, Sarasota, FL. I would like to thank Linda Ozin and Scott Mintz for participating as subjects, and Allison B. Sekuler for commenting on an earlier draft of the manuscript. This research was supported by National Science and Engineering Research Council of Canada Grant OGO042133. Correspondence should be addressed to P. J. Bennett, Department of Psychology, University of Toronto, 100 St. George St., Toronto, ON, Canada M5S 1A1. $180^{\circ}$ relative phase shift was visible. Figure 1 shows phase-reversal (i.e., $180^{\circ}$ relative phase shift) thresholds for 2 observers, as measured by Bennett and Banks (1991) using Field and Nachmias's procedure. In this figure, the contrast of $2 f$ is represented as the length of a vector joining a point and the origin, and base phase is represented by the vector's angle. Alternatively, each combination of $2 f$ contrast and phase can be represented as the sum of orthogonal sine and cosine components. Notice that discrimination of a $180^{\circ}$ shift occurred when the change in either the cosine or the sine component exceeded some threshold. These results are not critically dependent on the contrast, orientation, or spatial scale of the stimulus (Bennett \& Banks, 1991), at least for patterns presented in the fovea. Qualitatively similar results are also obtained with peripherally viewed targets, although the ratio of $90^{\circ}-270^{\circ}$ and $0^{\circ}-180^{\circ}$ thresholds varies significantly across the visual field and with stimulus orientation and spatial scale (Bennett \& Banks, 1987, 1991; Morrone, Burr, \& Spinelli, 1989).

The results shown in Figure 1 highlight an interesting fact about phase-reversal discrimination, namely that sensitivity for all $180^{\circ}$ shifts can be predicted from only two measurements (i.e., thresholds for $0^{\circ}-180^{\circ}$ and $90^{\circ}-270^{\circ}$ phase shifts). This simple empirical relation is not dependent upon any specification of the spatial features, or on the underlying neural mechanisms, used to discriminate phase shifts, and therefore it greatly simplifies the description of human performance in this phase-discrimination task. The current experiments examine whether this simple relation among phase-reversal thresholds extends to patterns other than $f+2 f$ gratings.

\section{Method}

\section{Stimuli}

The stimuli were vertical compound sine-wave gratings composed of two principal frequency components. They were generated on a Macintosh IIx microcomputer and passed through a 12-bit DAC and adjustable step attenuator to a Tektronix 608 CRT with P31 phosphor. The attenuator was adjusted so that the entire 12-bit DAC 


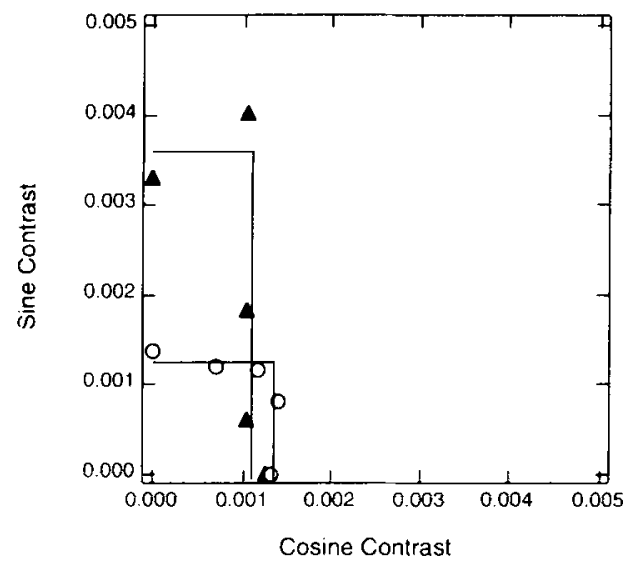

Figure 1. Examples of $180^{\circ}$ relative phase discrimination thresholds measured with $f+2 f$ compound gratings. The solid triangle and open circles show thresholds from different observers. For these measurements, the contrast of the fundamental was 0.2 and its spatial frequency was $2 \mathrm{cpd}$. Threshold is represented as the length of a vector joining each point to the origin, and base phase is represented as the vector's angle to the horizontal axis. Alternatively, each point can be considered as the vector sum of orthogonal cosine and sine components. Each point represents the threshold for a different $180^{\circ}$ shift: $0^{\circ}-180^{\circ}$ thresholds are plotted on the horizontal axis, $90^{\circ}-270^{\circ}$ thresholds on the vertical axis, and thresholds for other shifts lie in between. The solid lines show the best-fitting vertical and horizontal line segments for each data set.

range produced contrasts from 0 to 0.45 . Calibrations were performed with a spot photometer (Optikon, Model S2). The relationship between DAC input and CRT luminance was linearized in software. The frame rate was $100 \mathrm{~Hz}$ with 500 lines per frame. Mean luminance was $75 \mathrm{~cd} / \mathrm{m}^{2}$. The CRT was the only source of illumination in the testing room. An opaque circular mask that showed 7 cycles of the fundamental was placed over the CRT .

\section{Observers}

Three observers were tested. Observer P.J.B. was emmetropic; Observers S.M. and L.O. were corrected-to-normal myopes. All observers had 20/20 Snellen acuity and normal visual fields. Observers P.J.B. and L.O. received extensive practice ( $>10,000$ trials) prior to the beginning of the current experiments. Observer S.M. had approximately 2,000 practice trials.

\section{Procedure}

A 2-interval forced choice procedure was used. Trials were initiated by the observer. For Observers P.J.B. and L.O., each trial consisted of two 120-msec intervals marked by tones and separated by $240 \mathrm{msec}$. Observer S.M. was tested with intervals of $240 \mathrm{msec}$, separated by $480 \mathrm{msec}$. The average luminance was constant throughout the experiment.

There were three experimental conditions. In the compound detection condition, one interval contained a simple grating, given by Equation 1:

$$
L_{x}=L_{o}\left[1+\mathrm{C}_{f} * \cos (2 \pi f x)\right]
$$

the other contained a compound grating, described by Equation 2:

$$
L_{x}=L_{o}\left[1+\mathrm{C}_{f} * \cos (2 \pi f x)+\mathrm{C}_{n f} * \cos (2 \pi n f x-\Omega)\right],
$$

where $L_{0}$ is average luminance, $f$ and $n f$ are the spatial frequencies of the fundamental and harmonic, $\mathrm{C}$ is Michelson contrast, and $\mathbf{\Omega}$ is the relative phase of the harmonic. Observers were instructed to select the interval containing the compound grating. In the phase discrimination condition, both intervals contained compound gratings that differed by a $180^{\circ}$ relative phase shift. One interval contained a pattern defined by Equation 2, and the other interval contained the phase-shifted pattern:

$$
L_{x}=L_{o}\left[1+\mathrm{C}_{f} * \cos (2 \pi f x)+\mathrm{C}_{n f} * \cos (2 \pi n f x-\Omega-\pi)\right] .
$$

One pattern was arbitrarily designated as the target, and the observers were instructed to select the interval containing that pattern. Luminance profiles of some of the stimuli used in phasediscrimination conditions are shown in Figure 2. Finally, in the contrast-randomization condition, phase-discrimination thresholds were measured while pattern contrast was varied by multiplying the amplitudes of the grating components $\left(C_{f}\right.$ and $C_{n f}$ in Equation 3 ) by a contrast gain factor selected from a uniform distribution ranging between $\pm 0.2 \mathrm{log}$ units. A different gain factor was selected in each interval on every trial. This randomization procedure did not alter the ratio of the amplitudes of the frequency components, but it effectively removed overall pattern contrast as a cue for discrimination. The contrast-randomization conditions were identical to the phase discrimination in all other respects.

The observers were familiarized with the patterns prior to each experimental session. Auditory feedback indicated the accuracy of the response after each trial. The waveforms were displaced randomly within the circular mask in between intervals to ensure that the observers could not use average or local luminance cues to make discriminations. The observers were instructed to fixate the center of the CRT. Chin- and headrests were used to stabilize head position. Viewing was monocular through the natural pupil (approximately $4 \mathrm{~mm}$ in diameter) in all conditions.

The phase shift used in phase discrimination and contrast randomization conditions was always $180^{\circ}$. When the harmonic, $n f$, is less than $f$, a $180^{\circ}$ relative phase shift is identical to an absolute shift of the compound grating. Because the absolute phase was randomized across intervals, $180^{\circ}$ relative shifts were impossible to discriminate when $n f$ was less than $f$. We therefore measured phasediscrimination thresholds only for higher harmonics: The parameter $n$ in Equation 2 was an integer ranging from 2 to 8 .

Contrast was varied according to a three-down/one-up staircase procedure. The staircase step size was initially $0.06 \mathrm{log}$ units, decreased to $0.03 \log$ units after the 4 th reversal, and it was decreased to a final value of $0.015 \mathrm{log}$ units after the 6 th reversal. A staircase ended after 20 reversals, and threshold was defined as the mean of the final 18 reversal points. The results from at least three staircases were averaged to yield the final estimates of threshold and standard error.

\section{RESULTS}

\section{Compound Detection and Phase Discrimination Thresholds}

The initial experiments on the effects of frequency separation on phase sensitivity were essentially replications of work reported previously by Lawden (1983). Figures 3 and 4 show $0^{\circ}-180^{\circ}$ and $90^{\circ}-270^{\circ}$ thresholds for 2 observers with fundamental frequencies of 0.5 and $2 \mathrm{cpd}$. Fundamental contrast was 0.3 for Observer P.J.B. (Figure 3) and 0.2 for Observer S.M. (Figure 4). Figure 5 shows thresholds from Observer L.O. measured with a fundamental frequency of $1 \mathrm{cpd}$ and contrasts of $0.3,0.1$, and 0.03. Thresholds in all conditions and for all observers were qualitatively similar, exhibiting a minimum at the second or third harmonic and rising monotonically and significantly at higher harmonics. The figures show all 
A

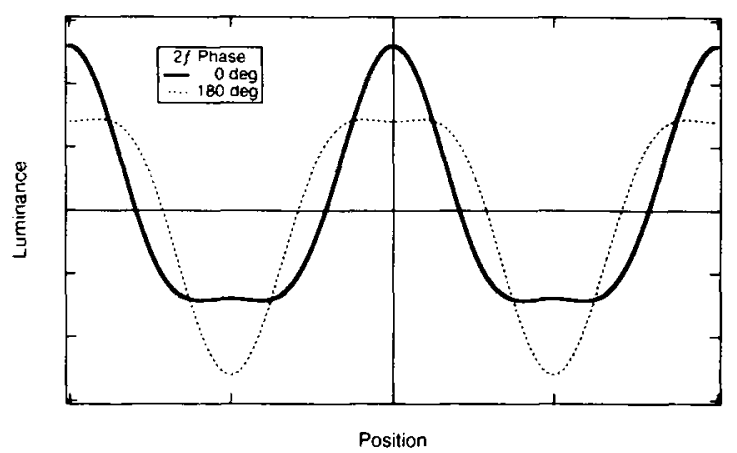

B

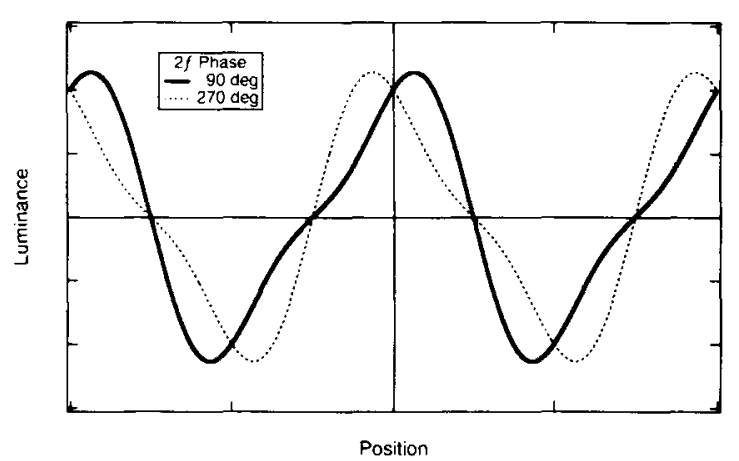

C

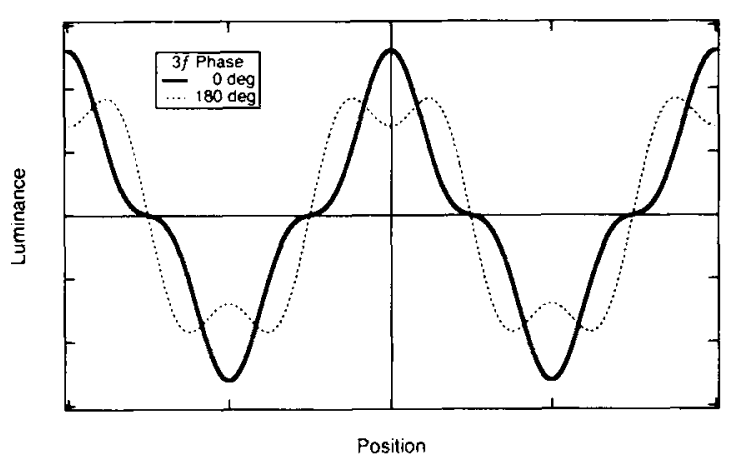

D

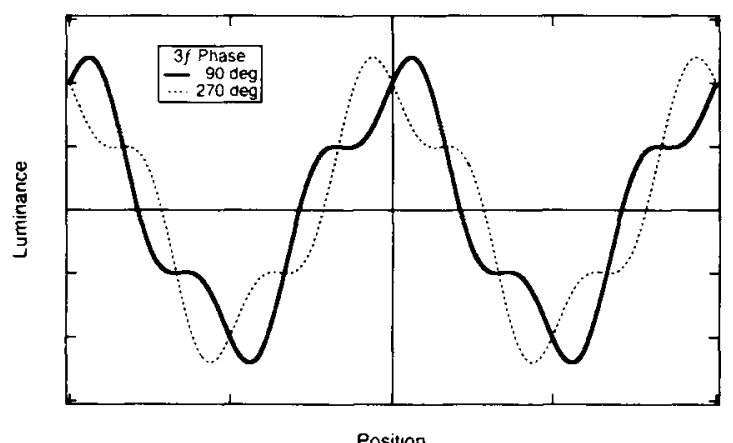

$E$

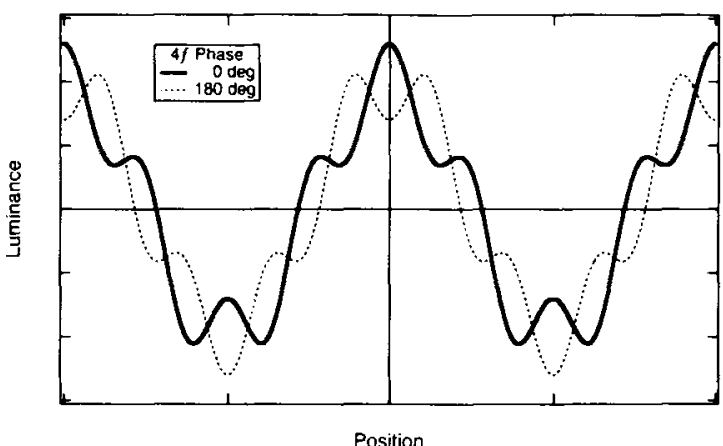

$F$

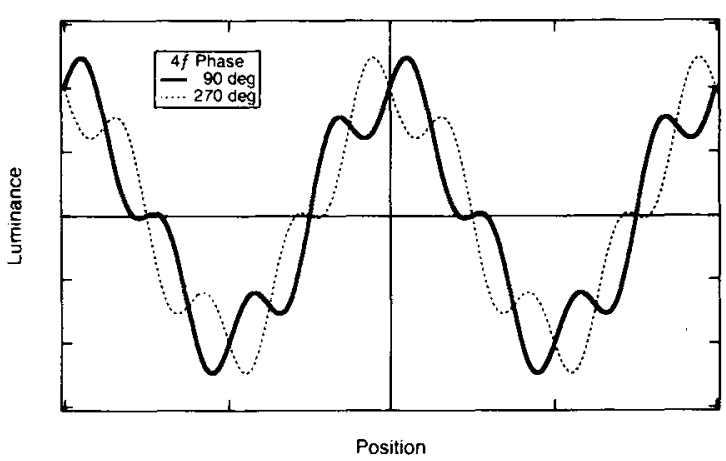

G

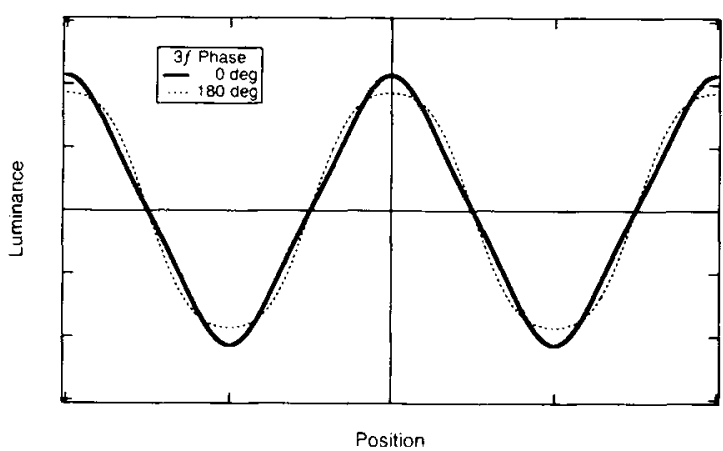

$\mathrm{H}$

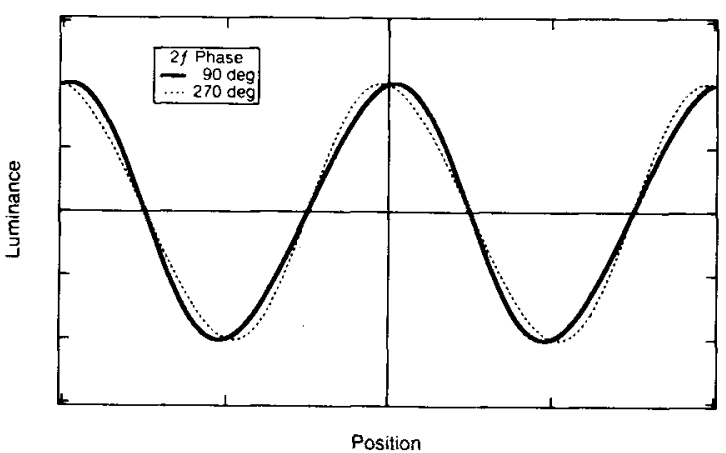

Figure 2. Luminance profiles of some of the waveforms used in phase discrimination conditions. The harmonic frequencies and relative phases are indicated in the legends. In panels $A-F$, the ratio of fundamental contrast divided by harmonic contrast is 3 . This ratio is much lower than the ratio of $f$ and $n f$ contrasts at discrimination threshold in real observers. The waveforms in panels $\mathbf{G}-\mathrm{H}$ were constructed with the ratio of fundamental and harmonic contrasts set to 15 ; they are better approximations of the luminance profiles of the stimuli at discrimination threshold. Panels A, B: $f+2 f$ waveforms. Panels C, D: $f+3 f$ waveforms. Panels E,F: $f+4 f$ waveforms. Panels G, H: $f+2 f$ and $f+3 f$ waveforms. 


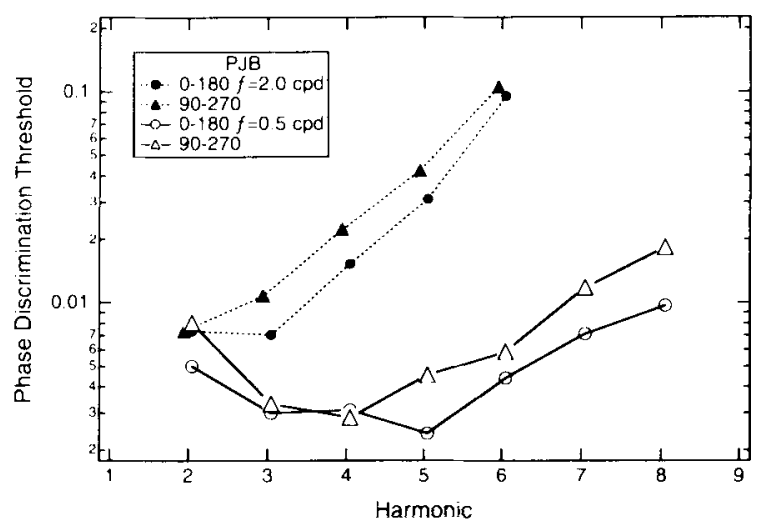

Figure 3. Discrimination thresholds for $0^{\circ}-180^{\circ}$ (circles) and $90^{\circ}-270^{\circ}$ (triangles) phase shifts for Observer P.J.B. Fundamental contrast was 0.3 . Fundamental frequency was 0.5 cpd (open symbols) or 2 cpd (filled symbols). Phase discrimination was not possible beyond the sixth and eighth harmonics in the 2- and 0.5-cpd conditions, respectively. Standard errors (not shown) were approximately 0.05 log units.

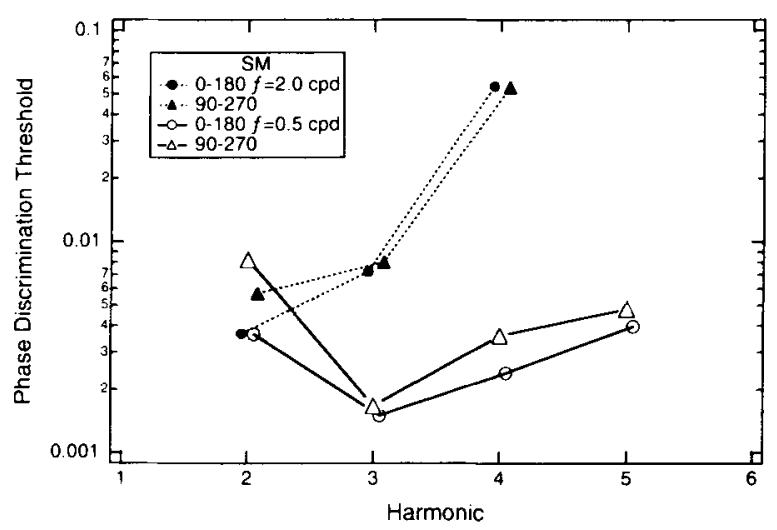

Figure $4.0^{\circ}-180^{\circ}$ and $90^{\circ}-270^{\circ}$ phase discrimination thresholds for Observer S.M. Fundamental contrast was 0.2. Symbol conventions are the same as in Figure 3. Phase discrimination was not possible beyond the fourth and fifth harmonics in the 2- and 0.5-cpd conditions, respectively. Standard errors (not shown) were approximately 0.05 log units.

harmonic combinations with which phase discrimination was possible; phase discrimination was impossible at higher harmonics. The harmonic range over which phase discrimination was possible was greatest when the fundamental was low in spatial frequency and high in contrast.

The variation in phase-discrimination thresholds across conditions is difficult to interpret because it might reflect differences in the visibility of the frequency components rather than phase sensitivity per se. In order to evaluate phase sensitivity directly, thresholds were normalized by computing the ratio of compound detection and phasediscrimination thresholds. Preliminary experiments revealed that compound detection thresholds measured with harmonics added in $0^{\circ}$ and $180^{\circ}$ relative phase did not differ (Figure 6A). Similarly, there was no difference between compound detection thresholds for $90^{\circ}$ and $270^{\circ}$ targets (Figure $6 \mathrm{~B}$ ). The $0^{\circ}-180^{\circ}$ and $90^{\circ}-270^{\circ}$ phasediscrimination thresholds were therefore normalized by dividing them by compound detection thresholds for the $0^{\circ}$ and $90^{\circ}$ phases, respectively.

The ratios of compound detection and phasediscrimination thresholds for 3 observers are shown in

\section{A}

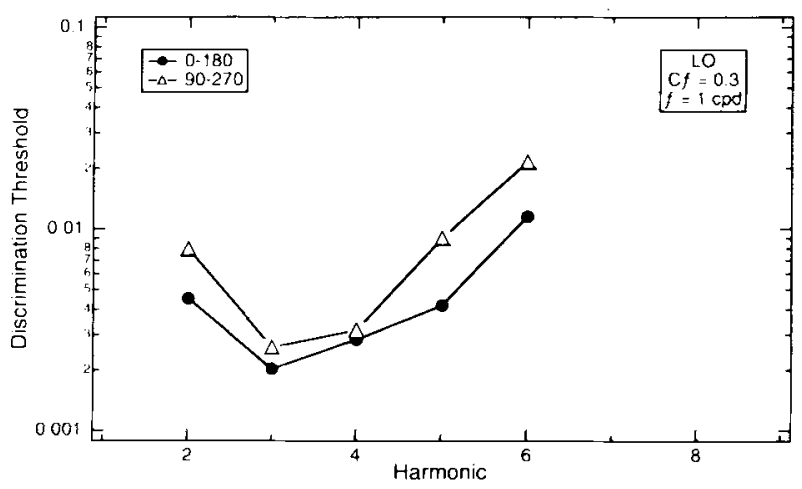

B

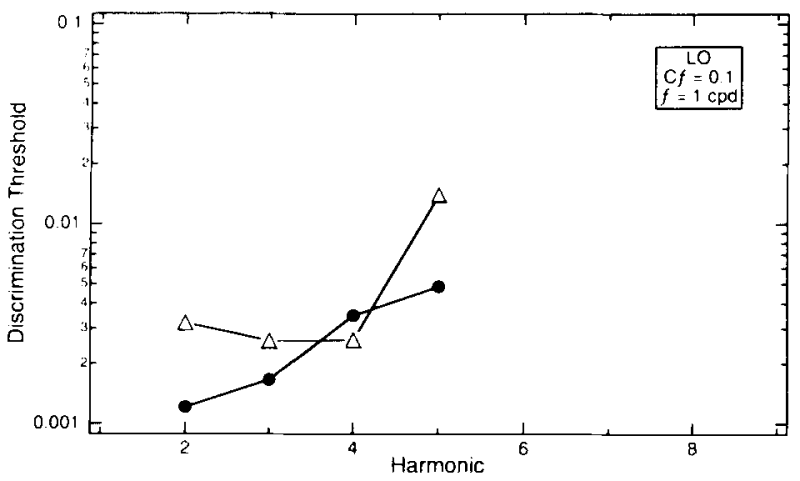

C

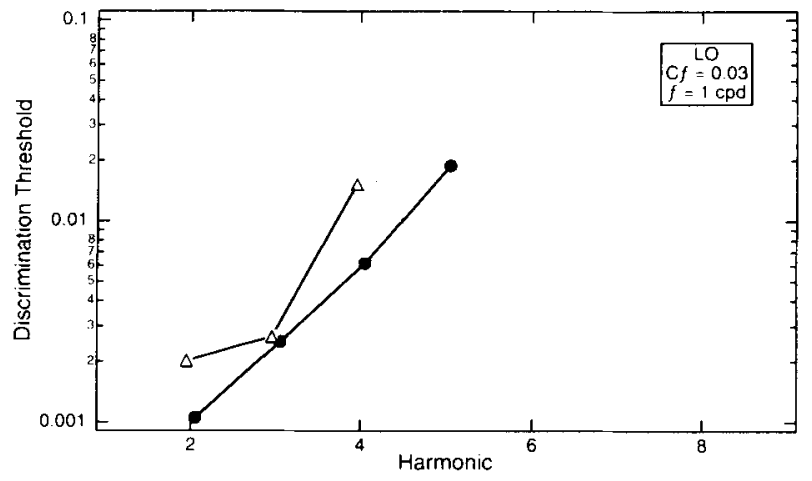

Figure 5. Discrimination thresholds for $0^{\circ}-180^{\circ}$ (filled circles) and $90^{\circ}-270^{\circ}$ (open triangles) phase shifts for Observer L.O. with fundamental contrasts $\left(C_{f}\right)$ of 0.3 (panel $A$ ), 0.1 (panel B), and 0.03 (panel C). The fundamental spatial frequency $(f)$ was 1 cpd. Note that the range over which phase-reversal discrimination is possible increases significantly at higher contrasts. Standard errors (not shown) were approximately $0.05 \log$ units. 
A

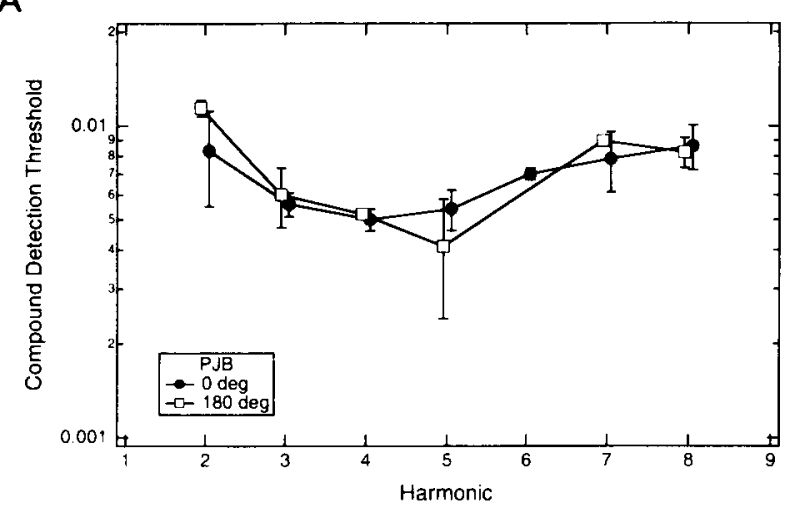

B

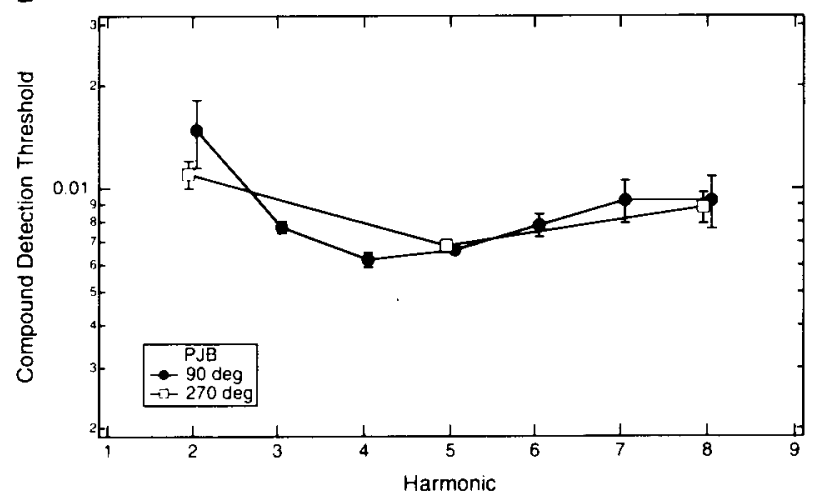

Figure 6. Compound detection thresholds for Observer P.J.B. Panel A: Thresholds obtained with harmonics added in $0^{\circ}$ (filled circles) and $180^{\circ}$ (open squares) phase. Panel B: Thresholds obtained with harmonics added in $90^{\circ}$ (filled circles) and $270^{\circ}$ (open squares) phase. Error bars indicate $\pm 1 S E$.

Figures 7-9. The solid lines in each figure are the predictions of a model that is outlined in the Discussion. Threshold ratios were generally greatest for $f+2 f$ and $f+3 f$ patterns and decreased sharply at higher harmonics. Hence, the increase in phase-reversal threshold that is evident in Figures 3-5 cannot be explained solely by reduced contrast sensitivity for the harmonic.

\section{Predictions of the Two-Channel Model}

Thresholds for $0^{\circ}-180^{\circ}, 90^{\circ}-270^{\circ}$, and other phase shifts were fit with the model

$$
T=\left[\frac{(a * b)^{k}}{[a * \sin (\Omega-\tau)]^{k}+[b * \cos (\Omega-\tau)]^{k}}\right]^{(1 / k)}
$$

where $T$ is threshold, $a, b, k$, and $\tau$ are free parameters, and $\Omega$ is the direction of the $180^{\circ}$ phase shift. When $k=2.0$, parameters $a, b$, and $\tau$ define an ellipse: $a$ and $b$ are the lengths of the axes, and $\tau$ is the orientation of the major axis. As the summation exponent, $k$, varies, the principle axes and orientation of the curve defined by Equation 4 remains unchanged, but the shape changes from a diamond $(k=1)$ to a rectangle (e.g., $k \geq 50$ ). In previous studies, phase-reversal thresholds for $f+2 f$ gratings have been well fit by a pair of horizontal and vertical line segments. To examine whether this result would be found with other two-component gratings, nonlinear regression was used to estimate parameters $a$ and $b$ while holding $k$ and $\tau$ constant at 50 and 90 , respectively. The regression results are presented in Tables 1 and 2 . In the tables, F1 and F2 refer to the spatial frequencies (cycles per degree) of the fundamental and harmonic. The contrast of $\mathrm{Fl}$ was 0.3 for Observers P.J.B. and L.O. and 0.2 for Observer S.M. Goodness-of-fit was evaluated with the chi-square statistic, and conditions in which the model was rejected $(p<.05)$ are indicated by asterisks. In all but two conditions, varying only parameters $a$ and $b$ resulted in satisfactory fits to the data.

Figure 10 shows the data from several conditions that were well fit by Equation 1 when $k$ and $\tau$ were held constant. Figure 11 shows the data from one of the conditions (Observer P.J.B., F1 = $2 \mathrm{cpd}, \mathrm{F} 2=12 \mathrm{cpd}$ ) in which the fit was poor. The thin lines in each figure are the regression curves computed with $k$ and $\tau$ held constant, and the thick line in Figure 11 is the curve computed when all four parameters were varied. Allowing $k$ and $\tau$ to vary did not improve the fits significantly, except in the two conditions marked in the tables. Casual inspection of Figures 10 and 11 might suggest an alternative to the model in Equation 4, namely that threshold is constant. A constant-threshold model predicts that the data shown in Figures 10 and 11 will be well fit by a circular arc. To test this idea, the best-fitting circles were computed for the 15 conditions listed in Tables 1 and 2 . The constant-threshold model provided very poor fits to the data and was rejected (at the $p<.025$ or $p<.005$ levels) in all but two conditions. Two features of the data accounted for the failure of the constant-threshold model. First, the average ratio of $90^{\circ}-270^{\circ}$ and $0^{\circ}-180^{\circ}$ thresholds was significantly greater than $1.0[t(14)=$ $3.86, p<.005$ ]. Second, the summation exponent, $k$, was greater than the value of 2 predicted by the constantthreshold model. In regressions where $\tau$ was constant and $a, b, k$ were allowed to vary, the median summation exponent was 14 (range $=2.1-60$ ), and a $t$ test on the logtransformed exponents indicated that the average summation exponent was significantly greater than $2[t(14)=$ $4.75, p<.001$ ]. I also applied the constant-threshold model to foveal and peripheral phase-reversal thresholds reported previously by Field and Nachmias (1984) and Bennett and Banks $(1987,1991)$ and found that the model fails to adequately fit those data, too.

The ratios of $90^{\circ}-270^{\circ}$ and $0^{\circ}-180^{\circ}$ thresholds are plotted in Figure 12 as a function of the harmonic content of the stimulus. All but three $90^{\circ}-270^{\circ} / 0^{\circ}-180^{\circ}$ ratios fell within a $0.3 \mathrm{log}$ unit range and they did not appear to vary systematically with the harmonic composition of the stimuli. 
A

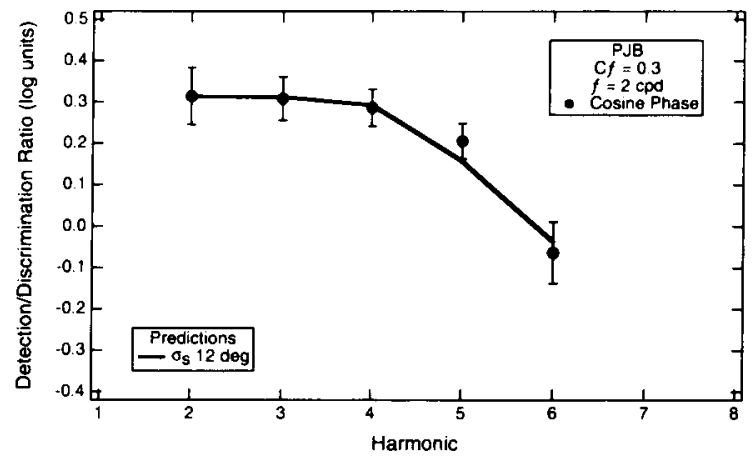

B

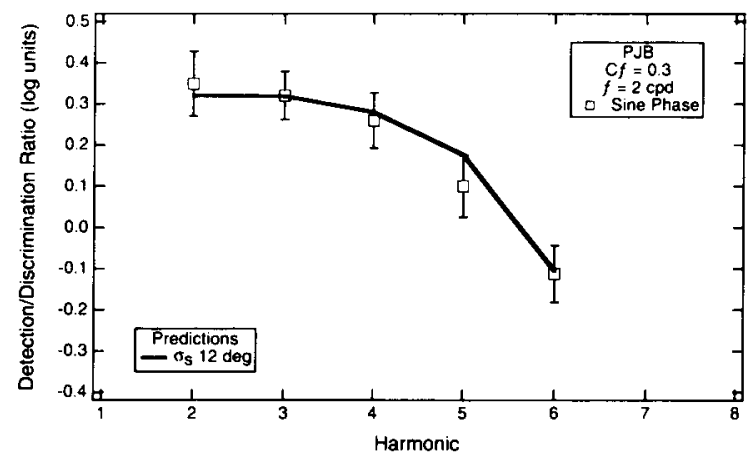

C

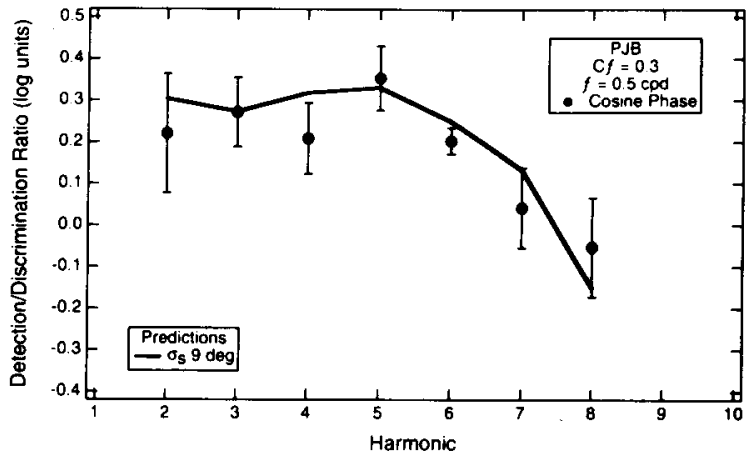

D

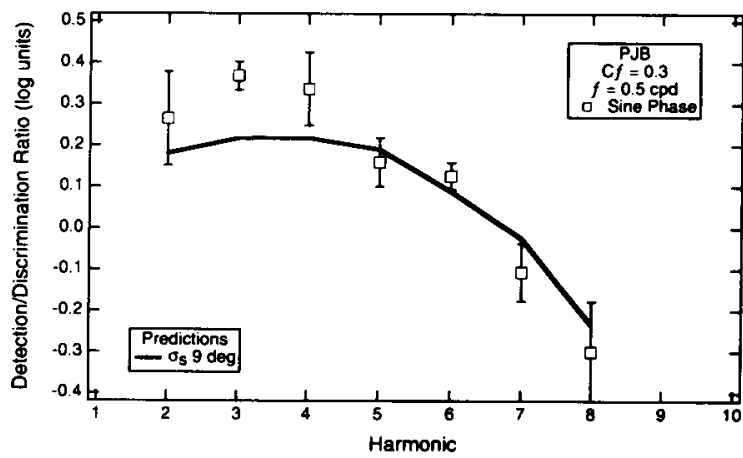

Figure 7. The ratio of compound detection thresholds divided by phase-discrimination thresholds for Observer P.J.B. plotted as a function of the harmonic number. The fundamental frequency $(f)$ and contrast $\left(C_{f}\right)$ are indicated in the figure legends. The solid lines are the predictions of the two-channel model, which is outlined in the Discussion, for different amounts of position jitter expressed in terms of phase angle $\left(\sigma\right.$, in the legends). Ratios for the cosine (i.e., $\left.0^{\circ}-180^{\circ}\right)$ condition are indicated by the filled circles in panels $A$ and $C$. Ratios for the sine (i.e., $90^{\circ}-270^{\circ}$ condition) are indicated by the open squares in panels B and D. Error bars indicate $\pm 1 S E$.

\section{Effects of Contrast Randomization}

$180^{\circ}$ phase shifts alter the peak-to-trough amplitude of $f+3 f$ and $f+5 f$ gratings. $180^{\circ}$ phase shifts do not alter the peak-to-trough amplitude of $f+2 f, f+4 f$, and $f+$ $6 f$ gratings, but they do change peak luminance. One way to examine whether observers are basing their discriminations on these cues is to measure thresholds while randomizing overall pattern contrast. Bennett and Banks (1991) have shown that randomizing overall pattern contrast has no effect on $180^{\circ}$ phase discrimination thresholds with $f+2 f$ gratings, so the effects of contrast randomization were examined only in patterns containing a third or higher harmonic. The $f$ contrast (prior to multiplication by the random gain factor) was set to 0.2 , rather than 0.3 , so that the maximum possible contrast (after multiplication by the random gain factor) fell within the display's linear range. It became difficult to discriminate phase shifts with $f+6 f$ gratings at this lower $f$ contrast, so thresholds were measured only with $f+3 f, f+4 f$, and $f+5 f$ patterns. The average difference between fixed and random contrast thresholds was less than $0.1 \mathrm{log}$ units. In control experiments, we measured the effect of contrast randomization in a contrast discrimination task. Randomizing contrast over a \pm 0.2 log unit range increased contrast discrimination thresholds by $0.3 \log$ units. Hence, the failure to obtain a significant effect of contrast varia- tion on phase discrimination thresholds was probably not caused by using an insufficient randomization range.

\section{DISCUSSION}

Field and Nachmias (1984) first reported that phasereversal discrimination in $f+2 f$ compound gratings occurs when either the cosine or the sine component of the phase shift exceeds some threshold. This result is virtually independent of the spatial frequency and contrast of the fundamental, at least for foveal targets (Bennett \& Banks, 1987, 1991). The current investigation demonstrates that similar results are obtained with other twocomponent gratings. With fundamentals of $0.5-2 \mathrm{cpd}$ at moderate contrasts, the harmonic bandwidth of phase discrimination is approximately two to three octaves. The bandwidth appears to increase slightly at lower fundamental frequencies and at higher fundamental contrasts. However, in all conditions in which discrimination was possible, phase reversals were discriminated whenever the change in the cosine or sine component of the phase shift exceeded some threshold. Furthermore, the ratio of $90^{\circ}-270^{\circ}$ and $0^{\circ}-180^{\circ}$ thresholds varies over only 0.3 $\log$ units across all conditions. Thus, the current findings demonstrate that it is possible to estimate phase-reversal thresholds with reasonable accuracy from only two mea- 
A

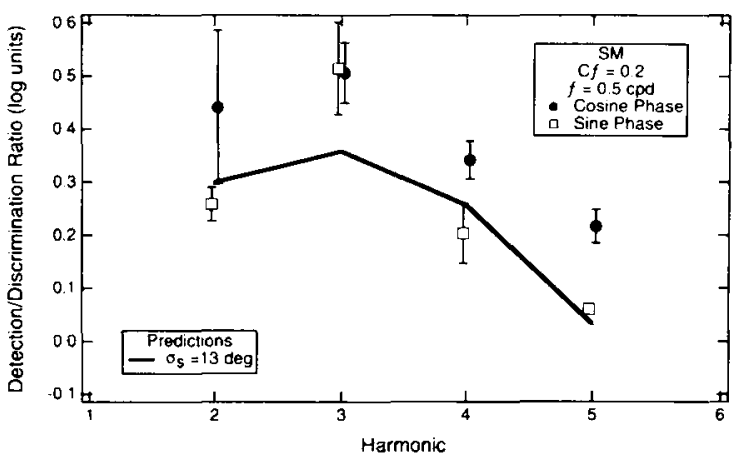

B

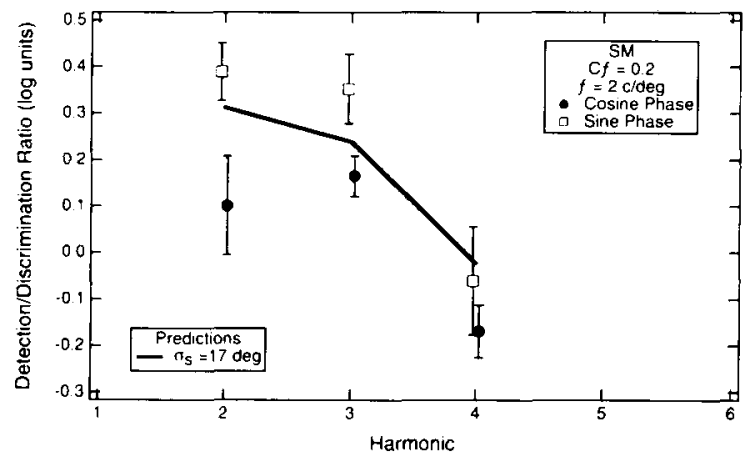

Figure 8. The ratio of compound detection thresholds divided by phase-discrimination thresholds for Observer S.M. plotted as a function of the harmonic number. The contrast of the fundamental contrast $\left(\mathrm{C}_{f}\right)$ was 0.2 . The solid lines are the predictions of the twochannel model for different amounts of position jitter expressed in terms of phase angle ( $\sigma_{2}$ in the legends). Ratios for the cosine (i.e., $0^{\circ}-180^{\circ}$ ) condition are indicated by the filled circles and those for the sine (i.e., $90^{\circ}-270^{\circ}$ ) condition by the open squares. Panel $\mathrm{A}$ : Fundamental frequency $=0.5 \mathrm{cpd}$. Panel B: Fundamental frequency $=2$ cpd. Error bars indicate $\pm 1 S E$.

surements (i.e., thresholds for $0^{\circ}-180^{\circ}$ and $90^{\circ}-270^{\circ}$ shifts).

Lawden (1983) first demonstrated that $0^{\circ}-180^{\circ}$ phase shifts between two gratings were discriminable provided that the frequencies were within approximately two octaves of each other. The current experiments replicate this finding and extend it to other $180^{\circ}$ shifts. It should be stressed that both frequency components were clearly visible in all phase-shift conditions, so the failure to discriminate shifts between frequencies that differed by more than two to three octaves was not caused by an inability to detect the harmonic. One interpretation of the discrimination failure is that observers perceived far-apart frequencies as two separate sinusoidal gratings instead of a single, fused pattern, and that such perceptual independence reflects the fact that phase-encoding mechanisms integrate information over only a limited range of spatial scales. Interpreted in this fashion, the current results are in good agreement with the findings of Thomas (1989), who, in several tasks, obtained converging evidence that suprathreshold gratings separated by more than two to two-and-a-half octaves are processed by independent mechanisms. A different interpretation of this result is outlined later in the Discussion.

\section{Phase Encoding Is Inefficient}

A constant-threshold model fails to account for phasereversal discrimination thresholds. This finding has important theoretical implications. Bennett and Banks (1991) used an ideal observer developed by Geisler (1984; Geisler \& Davila, 1985) to show that the amount of information available (at the level of photon absorption in the receptor lattice) in a phase-reversal discrimination task is independent of base phase. In other words, the amount of available information is identical for $0^{\circ}-180^{\circ}$, $90^{\circ}-270^{\circ}$, and any other $180^{\circ}$ relative phase shift. Hence, real observers ought to have equal thresholds for all $180^{\circ}$ shifts if they used the available information efficiently. The fact that they are not equal cannot be explained on the basis of some shifts' being intrinsically more difficult to detect than others. Instead, differences in phase sensitivity must reflect inefficiencies in phase-encoding mechanisms.

\section{Local Contrast and Spatial Features}

One way of modeling this inefficient processing is to suppose that phase discrimination is based on local analyses of only a small set of spatial features (Badcock, 1984a, 1984b; Hess \& Pointer, 1987; Watt, 1985). Badcock (1984a, 1984b), for example, has suggested that changes in relative luminance (i.e., local contrast) of conspicuous pattern features may serve as a discrimination cue under some conditions. In most of Badcock's experiments, the features used to compute local contrast were adjacent luminance maxima and minima, so it is reasonable to assume that such features are used in the current tasks, too. In many of the conditions studied in the current paper, the contrast of the fundamental was approximately 1.5-2 log units greater than the contrast of the shifted harmonic at discrimination threshold. Thus, at phase-reversal threshold, the luminance maxima and minima corresponded to the peaks and troughs of the compound gratings (see Figure 2, panels G-H). Because the local contrast between these features was randomized in the contrast-randomization conditions, the local contrast model predicts that discrimination thresholds should increase in those conditions. Instead, contrast randomization had no systematic effect on phase-discrimination thresholds. It seems, therefore, that the observers did not utilize overall or local contrast cues when discriminating $180^{\circ}$ phase shifts (see also Hess \& Pointer, 1987). As an alternative to the local contrast model, Hess and Pointer suggested that changes in luminance gradients are used to discriminate some phase shifts. For the stimuli used in our experiments, changing contrast alters the luminance gradients in the waveforms, so the results of the contrastrandomization experiment, and of a similar experiment reported previously (Bennett \& Banks, 1991), suggest that 
A

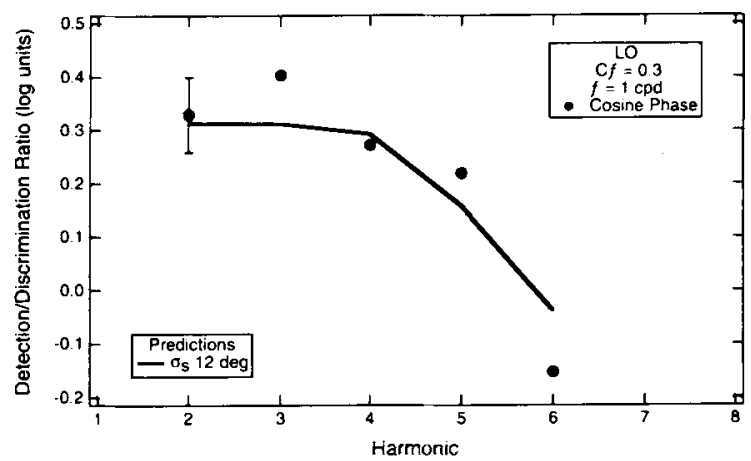

B

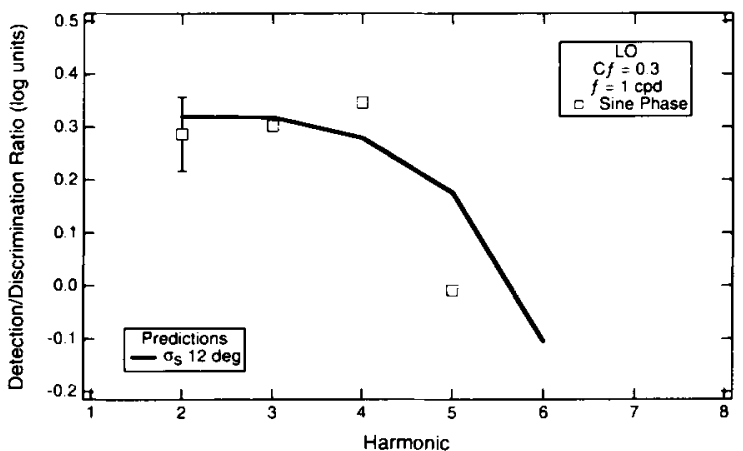

C

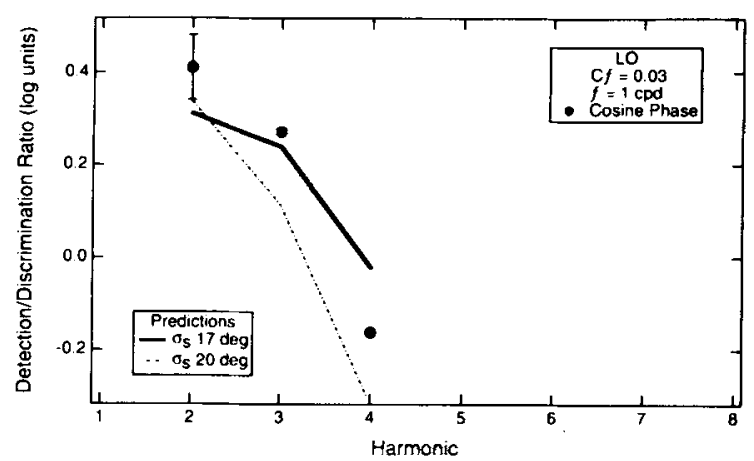

D

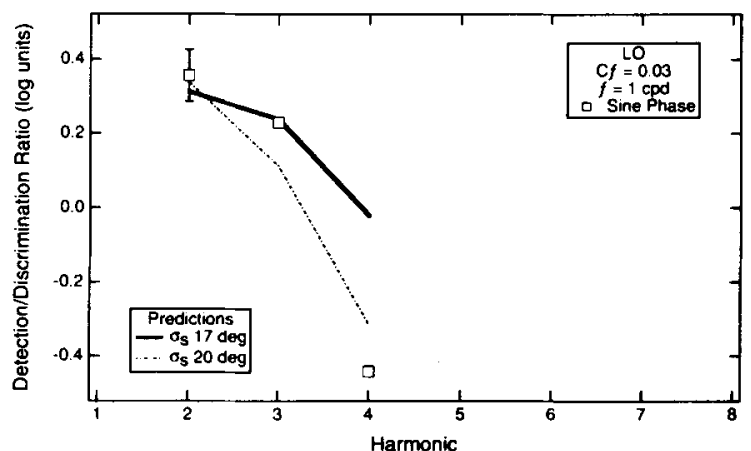

Figure 9. The ratio of compound detection thresholds divided by phase-discrimination thresholds, measured at two fundamental contrasts, plotted as a function of the harmonic number. The observer was L.O. Fundamental frequency $(f)$ was 1 cpd; fundamental contrast $\left(C_{f}\right)$ is indicated in the figure legends. The solid lines are the predictions of the two-channel model for different amounts of position jitter expressed in terms of phase angle ( $\sigma_{z}$ in the legends). Ratios for the cosine (i.e., $0^{\circ}-180^{\circ}$ ) condition are indicated by the filled circles in panels $A$ and $C$. Ratios for the sine (i.e., $90^{\circ}-270^{\circ}$ condition) are indicated by the open squares in panels $B$ and $D$. The error bar in each panel indicates \pm 1 average $S E$.

Table 1

Results of Nonlinear Regression for Observer P.J.B.

\begin{tabular}{lcllccrr}
\hline $\begin{array}{c}\text { F1 } \\
\text { (cpd) }\end{array}$ & $\begin{array}{c}\text { F2 } \\
\text { (cpd) }\end{array}$ & \multicolumn{1}{c}{$a$} & \multicolumn{1}{c}{$b$} & \multicolumn{1}{c}{$\boldsymbol{c}$} & \multicolumn{1}{c}{$k$} & \multicolumn{1}{c}{$\chi^{2}$} & $d f$ \\
\hline 0.5 & 4.0 & 0.009 & 0.025 & 90 & 50 & 19.4 & 12 \\
0.5 & 3.5 & 0.007 & 0.011 & 90 & 50 & 7.5 & 8 \\
0.5 & 2.5 & 0.0025 & 0.0058 & 90 & 50 & 8.7 & 8 \\
2 & 12 & 0.086 & 0.105 & 90 & 50 & $25.8 *$ & 11 \\
& & 0.112 & 0.147 & 122 & 1.1 & 11.3 & 9 \\
2 & 10 & 0.034 & 0.039 & 90 & 50 & 6.3 & 6 \\
2 & 8 & 0.015 & 0.021 & 90 & 50 & 4.2 & 5 \\
2 & 6 & 0.008 & 0.01 & 90 & 50 & 4.7 & 5 \\
2 & 4 & 0.006 & 0.009 & 90 & 50 & 8.4 & 5
\end{tabular}

*Condition in which the model was rejected.

Table 2

Results of Nonlinear Regression for Observers S.M. and L.O.

\begin{tabular}{|c|c|c|c|c|c|c|c|c|}
\hline Observer & $\begin{array}{c}F 1 \\
\text { (cpd) }\end{array}$ & $\begin{array}{c}\text { F2 } \\
\text { (cpd) }\end{array}$ & $a$ & $b$ & $\tau$ & $k$ & $x^{2}$ & $d f$ \\
\hline S.M. & 2 & 8 & 0.051 & 0.059 & 90 & 50 & 2.2 & 5 \\
\hline S.M. & 2 & 6 & 0.006 & 0.007 & 90 & 50 & 7.8 & 5 \\
\hline S.M. & 2 & 4 & 0.004 & 0.006 & 90 & 50 & 8.4 & 5 \\
\hline L.O. & 1 & 5 & 0.004 & 0.007 & 90 & 50 & 15.4 & 9 \\
\hline \multirow[t]{2}{*}{ L.O. } & 1 & 4 & 0.002 & 0.003 & 90 & 50 & $21.9^{*}$ & 8 \\
\hline & & & 0.003 & 0.003 & 71 & 3 & 11.0 & 6 \\
\hline L.O. & 1 & 3 & 0.002 & 0.002 & 90 & 50 & 11.0 & 8 \\
\hline
\end{tabular}

*Condition in which the model was rejected. phase-reversal discrimination is not based on changes in the magnitude of the maximum luminance gradient either.

The utility of other cues was also examined. Luminance profiles of the stimuli were created in software and, after subtracting the $\mathrm{dc}$ component from the waveforms, the centroid, standard deviation, equivalent width, skewness, and kurtosis of each positive lobe were calculated. The working hypothesis used to evaluate the utility of these measures was that, at threshold, changes in a statistic should be constant across conditions. For example, if $90^{\circ}-270^{\circ}$ shifts were discriminated on the basis of shifts in the position of the centroid in a single bright bar, then the magnitude of the centroid shift should be similar for $f+2 f, f+3 f$, and $f+4 f$ gratings. Thus, transforming $90^{\circ}-270^{\circ}$ phase-reversal thresholds shown in Figures 3-5 into measures of centroid displacement should cause the thresholds to be similar for all compound gratings. However, considerable variability across conditions remained even after transforming phase-reversal thresholds into these measures: In no case was the range of transformed thresholds significantly less than the range of original thresholds shown in Figures 3-5. According to this criterion, therefore, little is gained by expressing phasereversal thresholds in terms of changes in spatial features. 
A

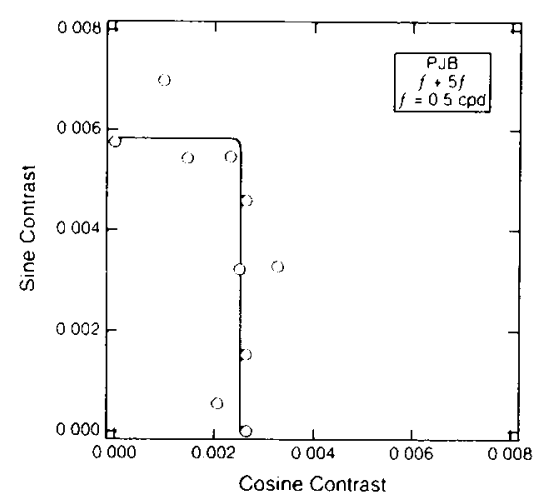

B

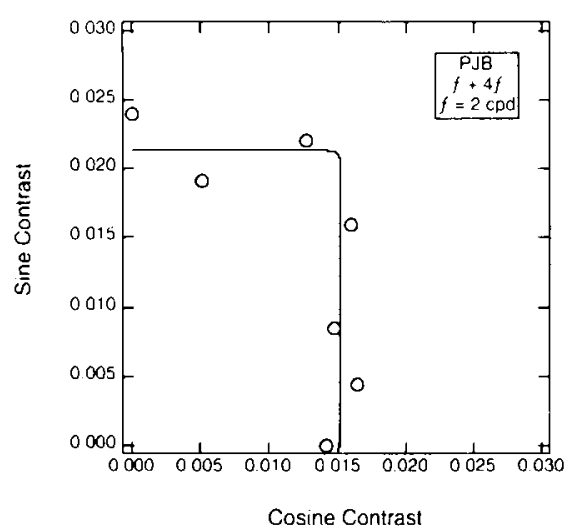

C

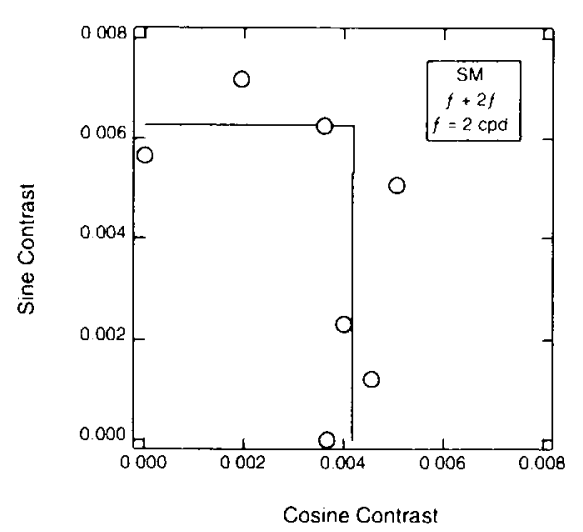

D

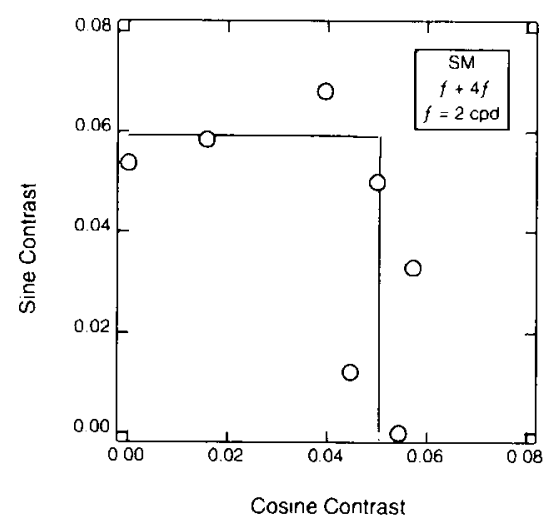

Figure 10. Typical discrimination thresholds for $180^{\circ}$ shifts measured for different harmonic combinations and plotted as in Figure 1 . The solid lines in each panel show the best-fitting curve (Equation 4) when $k$ and $\tau$ are fixed at 50 and 90 , respectively. The deviations from the best-fitting curve are not statistically significant. The slight distortion at the upper-right corner of each curve is an artifact introduced by the graphing routines. Standard errors (not shown) are approximately $0.05 \log$ units. Panel A: Observer P.J.B., $f+5 f$, $f=0.5$ cpd. Panel B: Observer P.J.B., $f+4 f, f=2$ cpd. Panel C: Observer S.M., $f+2 f, f=2$ cpd. Panel D: Observer S.M., $f+$ $4 f, f=2$ cpd.

\section{Predictions of a Two-Channel Model}

Of course, one could argue that the features that were analyzed were not appropriate for these tasks. One problem with local feature models is that it is difficult to specify, a priori, the features that are important for any particular phase discrimination task. One way to get around this problem is to suppose that phase discrimination is mediated by general-purpose spatial filters that operate only at specific positions within the stimulus. Bennett and Banks $(1987,1991)$ suggested that only even-symmetric mechanisms centered on the peaks and troughs of the fundamental and odd-symmetric mechanisms centered on the zero-crossings contribute to discrimination. This position restriction assumption was necessary in order to explain why $f+2 f$ phase-reversal thresholds fall along vertical and horizontal contours when plotted as in Figure 1. The assumption leads to the incorrect prediction that $90^{\circ}-270^{\circ}$ shifts in $f+3 f$ and $f+5 f$ gratings should be indiscriminable, but a simple modification of the model renders it consistent with the current findings. Specifically, the revised position restriction assumption is that even- and oddsymmetric mechanisms centered only on the peaks and troughs of $f$ are used to discriminate phase shifts. It is straightforward to show that this model correctly predicts that thresholds for $180^{\circ}$ relative phase shifts fall along vertical and horizontal contours when plotted as in Figure 1. Furthermore, with the additional assumption that there is some small error associated with the placement of even- and odd-symmetric filters on the peak of the fundamental, the model accounts for the finding that relative phase shifts are discriminable only within a two- to three-octave band of spatial frequency.

Monte Carlo simulations were performed to test the two-channel model (see the Appendix for details). The simulation used the responses of linear even- $\left(\mathbf{R}_{c}\right)$ and oddsymmetric $\left(\mathrm{R}_{\mathrm{o}}\right)$ filters centered on one peak of $f$. It was assumed that the even- and odd-symmetric filters were tuned to the spatial frequency of the phase-shifted harmonic. Responses $R_{e}$ and $R_{o}$ were squared and summed to provide a third response $\left(\mathbf{R}_{c}\right)$ that was a monotonic function of the harmonic's contrast and independent of its phase. The model's performance in compound detection and phase discrimination tasks was limited by three independent sources of noise. Gaussian noise was added sep- 


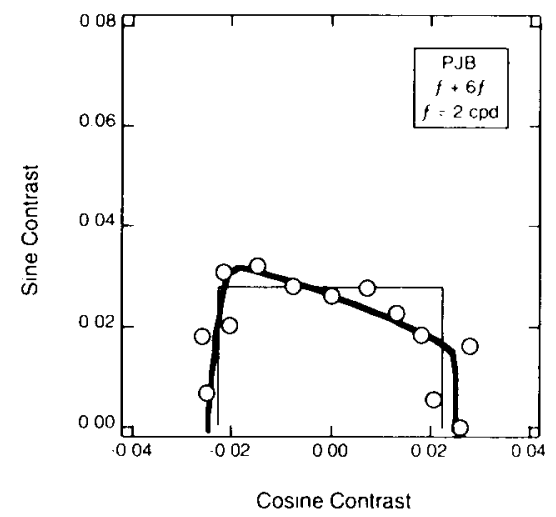

Figure 11. Discrimination thresholds for $180^{\circ}$ shifts measured with $f+6 f$ gratings in Observer P.J.B. and plotted as in Figure 1. These thresholds are one of two conditions that are not adequately fit by Equation 4 when $k$ and $\tau$ are fixed at 50 and 90 , respectively (thin line). The thick solid line shows the best-fitting curve obtained from Equation 4 when all parameters are allowed to vary. Fundamental frequency was 2 cpd. Standard errors (not shown) are approximately $0.05 \log$ units.

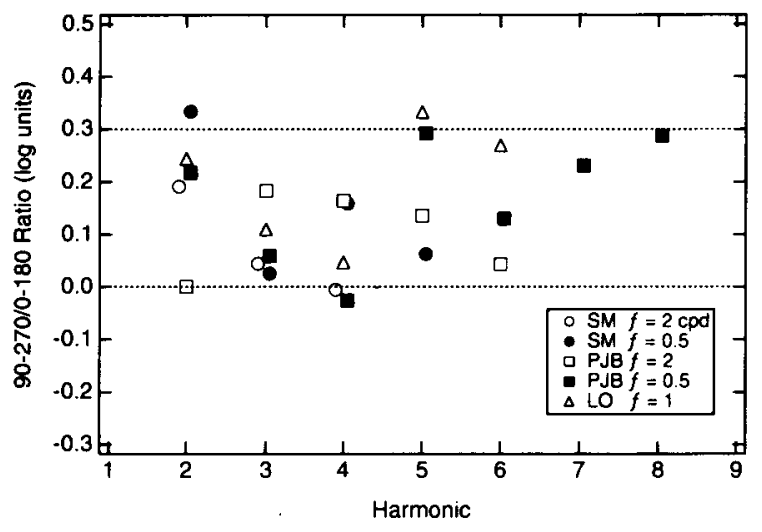

Figure 12. The ratio of $90^{\circ}-270^{\circ}$ thresholds divided by $0^{\circ}-180^{\circ}$ thresholds plotted as a function of the harmonic frequency. The ratios for 3 observers and fundamental frequencies $(f)$ are shown. Open circles: Observer S.M., $f=2$ cpd. Filled circles: Observer S.M., $f=0.5$ cpd. Open squares: Observer P.J.B., $f=2$ cpd. Filled squares: Observer P.J.B., $f=0.5$ cpd. Open triangles: Observer L.O., $f=1$ cpd.

arately to $R_{e}$ and $R_{0}$. These two noises restricted the detectability of the harmonic and were the principal factors governing sensitivity in phase-discrimination and compound detection tasks with $f+2 f$ and $f+3 f$ gratings.

The third noise that constrained sensitivity was the accuracy with which the peak of the fundamental was localized and, consequently, the placement of the even- and odd-symmetric filters. In the simulations, both the evenand the odd-symmetric filters were displaced from the fundamental's peak by a random amount drawn from a zeromean Gaussian distribution with a standard deviation of $\sigma_{\mathrm{s}}$. The even- and odd-symmetric filters were displaced by the same amount, but the size of the displacement changed on each interval of every simulated 2-IFC trial. With relatively low harmonics (i.e., $2 f$ and $3 f$ ), the position jitter had only a small affect on $R_{e}$ and $R_{0}$. At higher harmonics, however, even a small amount of position jitter introduced considerable variability in $R_{\boldsymbol{e}}$ and $\boldsymbol{R}_{\mathbf{0}}$, causing phase discrimination thresholds to increase significantly. Note, however, that the contrast response, $R_{c}$, is unaffected by position jitter and therefore could serve as a useful cue in compound detection tasks even at higher harmonics. The net effect of the position noise was therefore to increase phase discrimination thresholds, but not compound detection thresholds, at higher harmonics of $f$.

The lines in Figures 7-9 show the predicted ratios of compound detection and phase-discrimination thresholds. Different amounts of noise were required to fit the data obtained with the different fundamental frequencies; the standard deviations expressed in degrees of phase angle, $\sigma_{\mathrm{s}}$, are shown in the figure legends. An increase in fundamental frequency (Figures 7 and 8) and a decrease in fundamental contrast (Figure 9) both required increased position jitter in the model. These changes in the assumed internal position jitter are qualitatively similar to the results of Bradley and Skottun (1987), who found that vernier displacement thresholds for sine-wave gratings increased with increasing spatial frequency and decreasing contrast.

The two-channel model accounts for the main effects exhibited in Figures 7-9, lending support to the idea that the limited bandwidth of relative phase discrimination is caused by a failure to encode the position of $f$ accurately. One concern is whether the amounts of position jitter that were assumed in the model are in fact reasonable. These standard deviations are within a factor of 2 of the vernier acuities for sine-wave gratings reported by Bradley and Skottun (1987) and so are plausible estimates of internal position jitter. But these standard deviations are inconsistent with other previously reported findings. Specifically, these amounts of jitter lead to predictions of minimum discriminable phase angles that are larger than those found previously. Consider, for example, a condition in which the contrast of the phase-shifted harmonic is fixed at some suprathreshold value, and the task is to discriminate a change in relative phase angle. If the harmonic's contrast is high enough, the two-channel model predicts that phase-angle discrimination is limited solely by position noise, and discrimination threshold will therefore be proportional to the harmonic number. In the model used to generate the predictions shown in Figures 7C and 7D, position jitter equalled $12^{\circ}$ of the fundamental. This model would therefore predict that the minimum discriminable phase angles for $f+2 f, f+3 f$, and $f+4 f$ gratings would be approximately $24^{\circ}, 36^{\circ}$, and $48^{\circ}$, values that are higher than previous measurements. For example, Burr (1980) reported thresholds of $20^{\circ}-30^{\circ}$ for $f+3 f$ gratings (across a wide range of fundamental frequencies) when $f$ and $3 f$ contrasts were 0.3 and 0.1 . Badcock $(1984 \mathrm{~b})$ obtained thresholds of $7^{\circ}, 9^{\circ}$, and $12^{\circ}$ with $f+2 f, f+3 f$, and $f+4 f$ gratings (fundamental frequency and contrast were $1.7 \mathrm{cpd}$ and 0.44 , respectively). Thus, the amounts of po- 
sition jitter needed to account for the bandwidth of phasereversal discrimination (Figures 7-9) appear to be inconsistent with measurements of minimum discriminable phase angles in compound gratings.

The stimuli and methods used by Burr (1980) and Badcock (1984b) differed in several respects from those used here. In order to examine whether the two-channel model can be applied to a phase-angle discrimination task, another experiment was conducted on Observer P.J.B. The task was to discriminate a waveform defined by Equation 2 from the waveform

$$
\begin{aligned}
L_{x}=L_{o} & {\left[1+\mathrm{C}_{f} * \cos (2 \pi f x)\right.} \\
& \left.+\mathrm{C}_{n f} * \cos \left(2 \pi n f x-\Omega-\Omega_{s}\right)\right],
\end{aligned}
$$

and thus was similar to the tasks used by Badcock and Burr. In this experiment, the spatial frequency and contrast of the fundamental were $2 \mathrm{cpd}$ and 0.3 , respectively. The harmonic's base phase, $\Omega$, was $0^{\circ}$, and a staircase procedure adjusted the size of the phase shift, $\Omega_{s}$, to find the minimum discriminable phase angle. In all other aspects, this experiment was identical to the phasediscrimination task described in the Method section.

Discrimination thresholds obtained across a wide range of harmonic contrasts are shown in Figure 13. Consistent with the findings of Badcock (1984a) and Burr (1980), the minimum discriminable phase angle declines monotonically with increasing contrast. The lowest threshold is approximately $10^{\circ}$, which is close to the average value

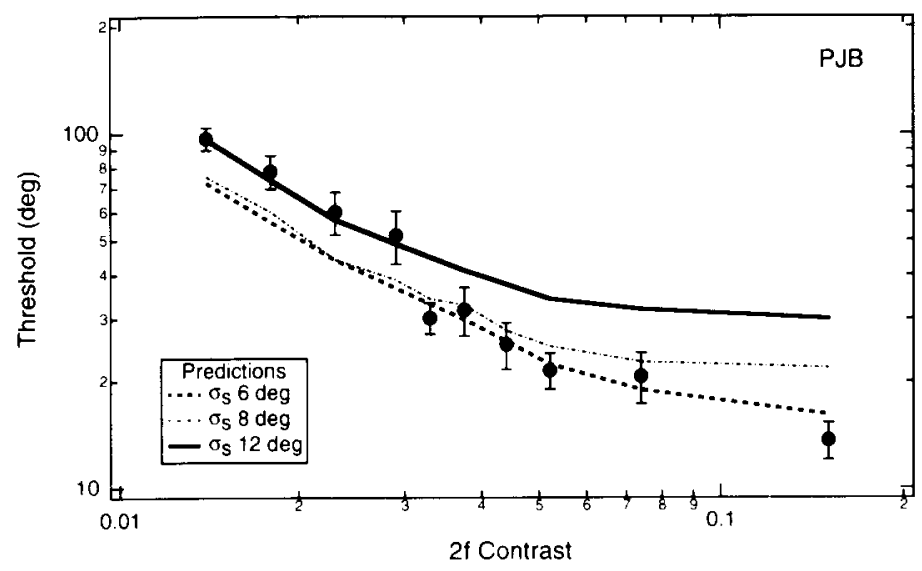

Figure 13. Minimum discriminable phase angles measured with $f+2 f$ gratings as a function of the contrast of $2 f$. The observer was P.J.B. The frequency and contrast of the fundamental were fixed at $2 \mathrm{cpd}$ and 0.3 , respectively. The curves indicate the predictions of the two-channel model for different amounts of position jitter expressed in terms of phase angle $\left(\sigma_{3}\right.$ in the figure legend). The error bars show $\pm 1 \mathrm{SE}$.

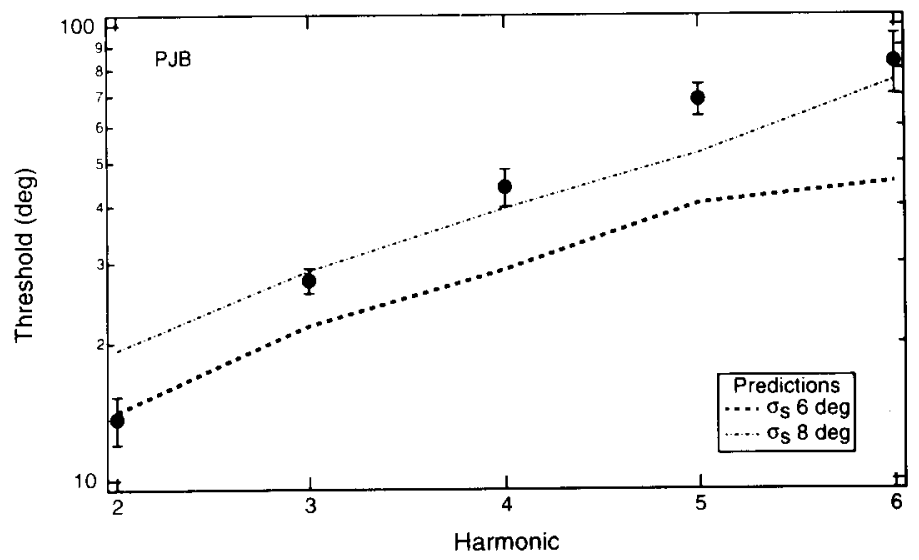

Figure 14. Minimum discriminable phase angles for different compound gratings. The observer was P.J.B. The frequency and contrast of the fundamental were fixed at 2 cpd and 0.3 , respectively. The harmonic's contrast was 0.15 . The curves indicate the predictions of the two-channel model for different amounts of position jitter expressed in terms of phase angle $\left(\sigma_{\mathrm{s}}\right.$ in the figure legend). The error bars show $\pm 1 S E$. 
obtained by Badcock (1984b) with similar stimuli. The same model used to generate the predictions shown in Figure 7 (panels A and B) was applied to this new task, and the predicted minimum discriminable angles are indicated by the solid line in Figure 13. Thresholds obtained at low $2 f$ contrasts are consistent with the predictions, but thresholds at high contrasts are much lower than the predicted values. The broken and dashed lines in Figure 13 show the predictions when the standard deviation of the position noise is decreased to phase angles of $8^{\circ}$ and $6^{\circ}$ : the model now accounts for thresholds at high contrasts but not at low contrasts. Figure 14 shows thresholds obtained with different compound gratings and the harmonic contrast set to 0.15 . Again, the data are well fit by the twochannel model only when the position noise is reduced to $6^{\circ}-8$. Similar results were obtained when the phaseangle discrimination experiments were replicated by $\mathrm{Ob}$ server S.M.

The data presented in Figures 13 and 14 show that the two-channel model accounts for phase-angle discrimination at low, but not high, harmonic contrasts. One interpretation of these results is that high-contrast harmonics reduce position jitter. There are at least two ways that this noise reduction could occur. First, even- and oddsymmetric filters could be placed on the peaks and troughs of the compound waveforms, which may be localized more accurately than the peaks and troughs of $f$ alone. Second, at sufficiently high contrasts, observers could use the harmonic itself, rather than the fundamental, as the spatial reference for positioning the even- and oddsymmetric filters. These hypotheses are currently being investigated.

In summary, the present experiments demonstrate that phase-reversal thresholds in two-component gratings follow a very simple pattern: Discrimination occurs whenever the cosine or sine component of the phase shift exceeds some criterion. This pattern of results holds regardless of the harmonic relationship of the components, provided that they are within two to three octaves of each other. These findings are consistent with a simple two-channel model that utilizes the responses of even- and oddsymmetric spatial filters (Bennett \& Banks, 1987, 1991; Field \& Nachmias, 1984).

\section{REFERENCES}

BADCOCK, D. R. (1984a). How do we discriminate relative spatial phase? Vision Research, 24, 1847-1858.

BADCOCK, D. R. (1984b). Spatial phase or luminance profile discrimination? Vision Research, 24, 613-624.

Bennett, P. J., * Banks, M. S. (1987). Sensitivity loss in oddsymmetric mechanisms and phase anomalies in peripheral vision. $\mathrm{Na}$ ture, 326, 873-876.

Bennetr, P. J., BANKs, M. S. (1991). The effects of contrast, spatial scale, and orientation on foveal and peripheral phase discrimination. Vision Research, 31, 1759-1786.

Bradley, A., Skottun, B. C. (1987). Effects of contrast and spatial frequency on vernier acuity. Vision Research, 27, 1817-1824.

BurR, D. C. (1980). Sensitivity to spatial phase. Vision Research, 20, 391-396.

Burr, D. C., Morrone, M. C., \& Sinelli, D. (1989). Evidence for edge and bar detectors in human vision. Vision Research, 29, 391-396.
Field, D. J., \& Nachmias, J. (1984). Phase reversal discrimination Vision Research, 24, 333-346.

GeISLER, W. S. (1984). Physical limits of acuity and hyperacuity. Joumal of the Optical Society of America A, 1, 775-782.

GeISLER, W. S., DA DILA, K. D. (1985). Ideal discriminators in spatial vision: Two-point stimuli. Joumal of the Optical Society of America A, 2, 1483-1497.

Hess, R. F., Pointer, J. S. (1987). Evidence for spatially local computations underlying discrimination of periodic patterns in fovea and periphery. Vision Research, 27, 1343-1360.

LAWDEN, M. C. (1983). An investigation of the ability of the human visual system to encode spatial phase relationships. Vision Research, 23, 1451-1464.

Morrone, M. C., Burr, D. C., Spinelli, D. (1989). Discrimination of spatial phase in central and peripheral vision. Vision Research, 29, 433-446.

OPPENheim, A. V., \& LIM, J. S. (1981). The importance of phase in signals. Proceedings of the IEEE, 69, 529-541.

Piotrowski, L. N., \& Camprell, F. W. (1982). A demonstration of the visual importance and flexibility of spatial-frequency amplitude and phase. Perception, 11, 337-346.

Tномаs, J. P. (1989). Independent processing of suprathreshold spatial gratings as a function of their separation in spatial frequency. Journal of the Optical Society of America A, 6, 1102-1111.

WATT, R. J. (1985). Structured representation in low-level vision. Na ture, 313, 266-267

\section{APPENDIX}

This Appendix describes the Monte Carlo simulations that were performed to evaluate the two-channel phase discrimination model outlined in the Discussion. The model utilizes the responses of linear even- and odd-symmetric spatial filters centered on the peak of the fundamental in a two-component grating. Furthermore, the model assumes that the filters responded only to the spatial frequency of the phase-shifted harmonic. If the relative phase of the harmonic is $\phi$, then the differential response of an even-symmetric filter produced by a $180^{\circ}$ relative phase shift is proportional to the absolute value of $\cos (\phi)$. Thus, the differential response is greatest for $0^{\circ}-180^{\circ}$ shifts and exactly zero for $90^{\circ}-270^{\circ}$ shifts. The differential response of an odd-symmetric filter is proportional to the absolute value of $\sin (\phi)$ and is therefore greatest for $90^{\circ}-270^{\circ}$ shifts and exactly zero for $0^{\circ}-180^{\circ}$ phase shifts. The two-channel model assumes that discrimination of $180^{\circ}$ phase shifts occurs when either the differential response of an even- or odd-symmetric filter exceeds some criterion, and therefore predicts that thresholds for $180^{\circ}$ relative phase shifts should fall along equal-cosine and equalsine contours when plotted as in Figure 1. One additional assumption is required to account for the finding that relative phase discrimination is possible only when the shifted components are within two to three octaves of each other. Specifically, it is assumed that position noise limits the precision with which the even- and odd-symmetric filters are centered on the peaks and troughs of $f$. Even a small amount of position noise significantly increases the variability of responses of even- and odd-symmetric filters to the higher harmonics of $f$, and therefore restricts relative phase discrimination to a relatively small range of spatial frequencies.

Simulations were performed as follows. The responses of the even- $\left(\mathbf{R}_{\boldsymbol{c}}\right)$ and odd-symmetric $\left(\mathbf{R}_{\mathbf{o}}\right)$ mechanisms located near one peak of the fundamental in the waveforms defined by Equations 2 and 3 were defined as:

$$
\begin{aligned}
& \mathbf{R}_{e}=C_{n f} \operatorname{Cos}\left\{2 \pi n f \mathrm{G}\left(\sigma_{\mathrm{s}}\right)-\mathbf{\Omega}\right\}+\mathrm{G}\left(\sigma_{\mathrm{e}}\right) \\
& \mathbf{R}_{\mathbf{o}}=\mathrm{C}_{n f} \operatorname{Cos}\left\{2 \pi n f \mathrm{G}\left(\sigma_{\mathrm{s}}\right)-\mathbf{\Omega}+\pi / 2\right\}+\mathrm{G}\left(\sigma_{\mathrm{o}}\right),
\end{aligned}
$$


where contrast, phase, and frequency of the phase-shifted harmonic are represented respectively by $\mathrm{C}_{n f}, \Omega$, and $n f$, and $\mathrm{G}\left(\sigma_{\mathrm{s}}\right)$, $\mathrm{G}\left(\sigma_{\mathrm{e}}\right)$, and $\mathrm{G}\left(\sigma_{\mathrm{o}}\right)$ are zero-mean Gaussian random variables. $\mathrm{R}_{\mathrm{e}}$ and $R_{0}$ were combined into a third response,

$$
\mathbf{R}_{c}=\mathbf{R}_{\mathrm{e}}^{2}+\mathbf{R}_{\mathrm{o}}^{2},
$$

which is monotonically related to the harmonic's contrast and independent of its phase. The contrast response, $R_{c}$, was useful only in compound detection conditions because harmonic contrast was unaffected by $180^{\circ}$ relative phase shifts. In compound detection conditions, responses $R_{e}, R_{o}$, and $R_{c}$ were computed for each of the waveforms described by Equations 1 and 2 . In phase discrimination conditions, $R_{e}, R_{o}$, and $R_{c}$ were computed for the waveforms described by Equations 2 and 3. Difference responses $-\Delta R_{e}, \Delta R_{0}$, and $\Delta R_{c}-$-were calculated by subtracting the response to the second waveform from the first, and appropriate decision rules were applied to each difference response separately. For example, if the task was to discriminate a $135^{\circ}-315^{\circ}$ relative phase shift, and the target was the $135^{\circ}$ phase pattern, the decision rules were

If $\Delta \mathrm{R}_{\mathrm{e}}<0$, then select Stimulus 1. Otherwise, select Stimulus 2;

If $\Delta R_{0}>0$, then select Stimulus 1. Otherwise, select Stimulus 2 .
It is important to stress that these decision rules were applied separately to the even-symmetric, odd-symmetric, and contrast responses. Such a decision strategy sometimes leads to conflicting responses, but there was no attempt to resolve such conflicts. By repeating the stimulus presentations, the percent-correct decisions based on each difference response could be estimated. Psychometric functions, based on at least 4,000 simulated trials, were generated for each difference response by varying parameter $\mathrm{C}_{n}$ in Equations 1-3, and the value yielding $80 \%$ correct responding was determined. The smallest value of $\mathrm{C}_{n f}$ was selected as the model's threshold for the particular condition being simulated. The model's unusual decision strategy is equivalent to assuming that real observers are not capable of combining the signals from the contrast and phase-encoding mechanisms, but instead monitor a single mechanism. Over time, it is assumed that observers learn which mechanism provides the most reliable signal for any given task.

Simulations were conducted on a Macintosh IIfx using doubleprecision (64-bit) floating point variables in the statistical and graphics environment of Igor (Version 1.25; WaveMetrics).

(Manuscript received July 31, 1991; revsion accepted for publication August 21, 1992.) 